\title{
Amazing autumn surgery behind the light
}

\author{
Martin Riegler
}

Published online: 4 September 2019

(c) Springer-Verlag GmbH Austria, part of Springer Nature 2019

Dear reader,

Welcome to this issue of European Surgery. After summer the autumn Indian Summer reaches the land. Hendrix plays, Jimmy saves (sozein) the blues as Cherokee Indians look out for the sky to follow the shine where the dog ran (milky way). This issue of the Journal includes stories within the focus of oesophageal and gastric surgery, the surgical management of diseases and disorders of the esophagus and the stomach, which impair the life quality and productivity of those affected. The spectrum of papers includes various reasons and causes for the development of diseases such as motility disorders of the esophagus, gastroesophageal reflux disease (GERD) and malignancies of the stomach. The authors and reviewers of the papers are to be congratulated for their outstanding work and for their passion to critically analyse their medical practice regarding the management of these diseases. Let us talk about food and greed, good indeed. Nutrition causes it all, the huge spectrum of hereditary genetics and para-genetics. Finally, the publisher and the editors are to be thanked for their outstanding support. Without their support our project ("what is the truth?") would not be possible. As a matter of fact, there exists a huge number of publishers, which compete for their acceptance, their markets and their profits, all around the globe. As another matter of fact, at the same time, millions of inhabitants of our beautiful planet turn around and face the anxiety, fear and psychological impairment caused and induced by the diagnosis of a disease and related to the diagnosis of inflammation, cancer and syndromes. This happens every day, every hour, every

Doz. Dr. M. Riegler $(\bowtie)$

Reflux Medical, Mariannengassse 10/9, 1090 Wien, Austria martin.riegler@refluxmedical.com second, every moment, whatever a moment means, senses and dilutes, sees and understands? How long lasts your moment for the assessment of a curious detail? Whom would you wish to see, if you had the choice: the oncologic surgeon, the manager of functional disorders of the gut (very GUT), the treatment seller for metabolic alterations or the psychologist? Which logos (i.e.collection) would you love to share if it were that you had a disease, a diagnosis related to the impairment of your life quality and well being? Thus, Aristotle found out that science happened to save (sozein) the phenomena. Therefore, whenever a doctor is going to see a patient, he or she marches in to save phenomena and to collect them for a diagnosis and to translate them into a therapy to save YOUR life (sozein ta phenomena). Can we be sure that all socialists know where the name describing their political and ethical idea, their moralistic attitude and method is coming from? Go ahead and ask socialists if they knew what sozein means? Conceptually, one should not like that such a fundamental element of our language is copied and superficially diluted within the power games of politics. But this observations proofs how superficial our reasoning and our critical approach became, as such developed the design of our modern society, which definitely is prone to any modern form of hidden despotism and ruling opinion. I think that we could do much better. What is your guess, dear reader? Do you think that all oncologists know where the name for their specialty is coming from? We have written about it many times in the recent issues of European Surgery. As such oncology is related to the story about Europa and the bull (red?) and to the symbolism of the zodiac clocks including the orientation of the pyramids, ancient temples, towers, churches and hospitals all over the globe, where the oceans stopped to wipe them out. Otherwise you may guess that it is not important to 
Fig. 1 The image cartoons the idea of the author, that all perceptions happen within the individual emotional matrix. Images a and $\mathbf{b}$ showing the beautiful landscape distal to the south end of the Traun lake, upper Austria, EU
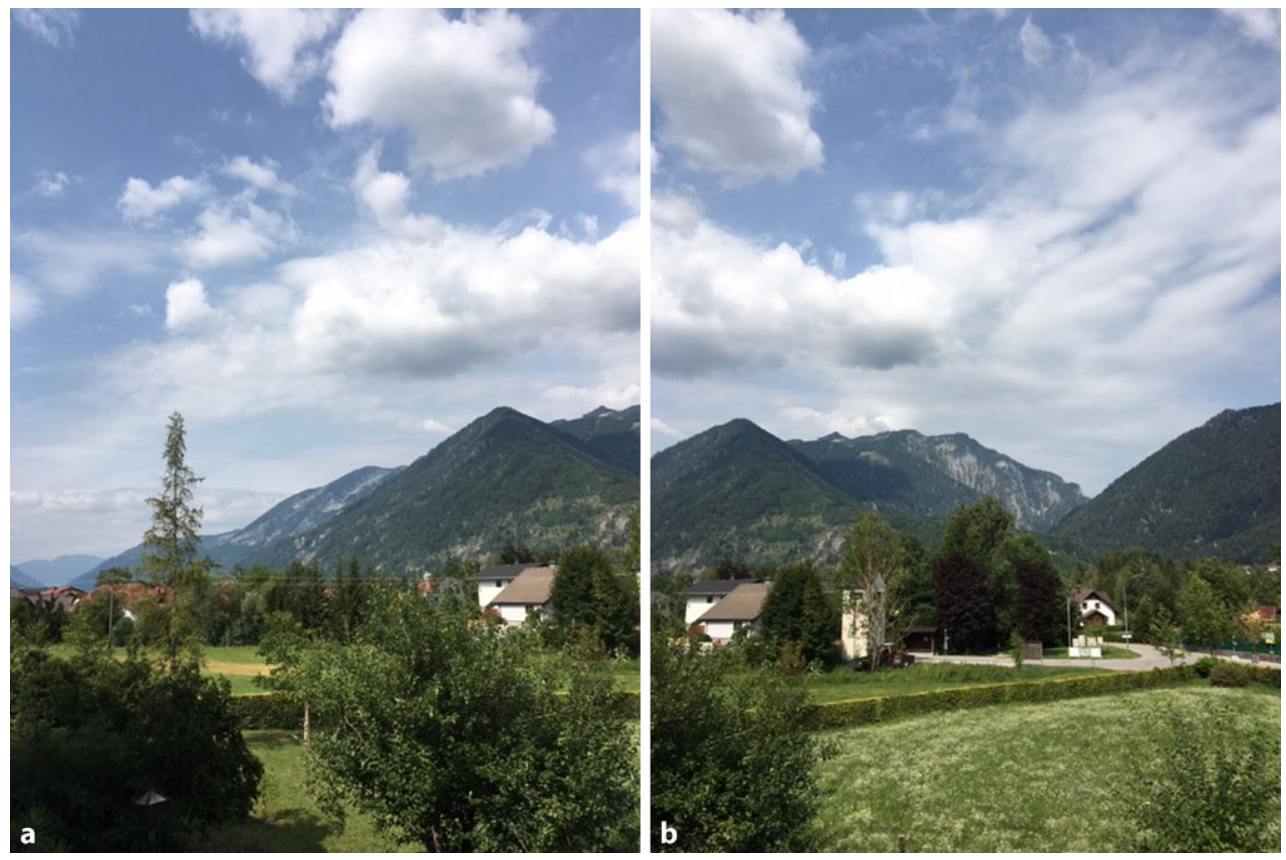

know the etymology of the instruments of your language, i.e. the translator of your science. Then you may be prone to be mislead by those, who know.

There is no festivity, no symposium, no celebration, no social event, no congress meeting without phagocytosis and pinocytosis. Therefore the gullet, the esophagus, the you-tube is fundamental for the performance of any celebration, festivity and cultural event, action and conduct of reasoning: "Should I go or should I stay (The Clash)?". Culture regulates digestion. Culture tells where, when, what to eat, drink and digest, and what we are not allowed to eat, drink and digest. In addition, culture regulates and controls, what we are allowed to say, argue and repeat. Sometimes, digestion dominates modern arts. Sometimes digestive motives orchestrate modern arts. Therefore the collection (logos) within this issue of Europe Surgery follows the motive related to those organs, which are essential for digestion, burb and postprandial complaints: the esophagus (the gullet) and the stomach (taking it all!). Ham let it be or not to be. At the end a culture may definitely digest its members, as previously described (i.e. history; $p<0.001$ ).

The author hopes that you had a great summer, that you found time and space, i.e. space time, to share with your friends, family and your outstanding ideas of interest. Hopefully you had great moments of enthusiasms and love and happiness and thankfulness and humility without fear, anger, hate, greed and fate. Hopefully you had time and space, i.e. space time quanta, for yourself, for your interests without serving the interests and powers of others. Thus the author hopes that you had single moments entirely for yourself. Do you know who you are? Did you learn who you are? Did you change? Did your family members change? Did your friends change? Did you find out who is your friend? Who knows your secrets and will not reveal them? What is going to happen after the holidays? Business and inflammatory responses as usual: hypocrites and parasites? Do you trust? Here we go and approach the fundamental sources and base, home basis for our reasoning and our perception.

Perception is essential for surgery. There is no surgery without perception. Therefore, the recent editorials aimed to ask how perception develops, works and how perception influences our daily work as surgeons, women, men, mothers and fathers. We came up with the idea, that we all are perceived as segments of the entire universe. Every one of us shares specific segments with others, the large majority remains hidden to us, i.e. the very truth. However, everyone is the centre of his or her individual universe. In addition, it seems impossible to know, see and understand it all, everything and the entire dimensions of the world. We are only allowed to deal with small little tiny segments of the universe. We only possess the translators for a limited numbers of phenomena (Fig. 1). From there we try to get an idea of the entire image. Theory creates the models. Using models we try to imagine how the entire universe may look like, behave and change. As such man developed models of understanding including myth, science, art and culture, which represent the intellectual base and platform for our societies.

Conceptually, myth and surgery share the fact, that both approaches serve a better understanding of the world and aim to translate perceptions into treatable signs. Furthermore, we found, that any interpretation of perceptions happens within the matrix of emotion, temper, mood and atmosphere. As such we and our knowledge are the results of the emotional matrix of 
Fig. 2 Anterior (a) and side aspect (b) of the so called Raurackl or Wolpertinger, which represents a human (embalmer) made fusion of different animals (bird, rabbit etc.). The image cartoons the idea that the google concept is unique as it fuses different aspects of our modern life including religions and various forms of industry, as described in the text
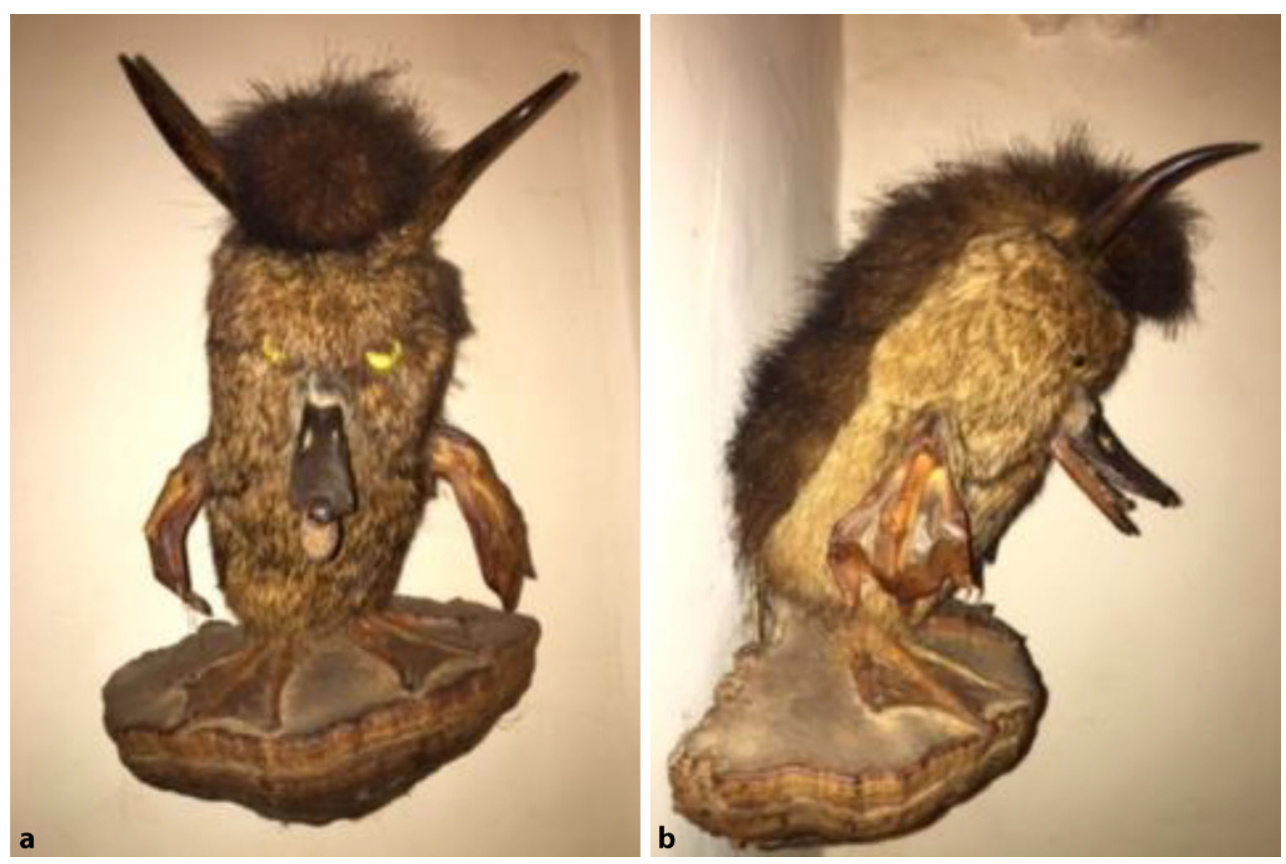

our universe. Remains to be questioned how this relates to our daily work (surgery).

Conceptually, our cultures are based upon religions and industries (technologies, robots etc.). Usually people lack the open minded approach and stay superficial. As such the leading opinion guidelines the people, the civilisations and the political and cultural behaviour of our societies. Going in line with the superficial approach religions and industries (science, economy, physics) are perceived as two different "things", "phenomena". Deeper reasoning may suggest that religions and industries may be two sides of a single coin. However, elementary and fundamental changes allow a completely different insight into this topic.

In the past 7000-8000 years or so, priests required industries for their power games, those working in the industries required religious support, and thus both phenomena interacted. Here I do not want to list all examples, but I am convinced that the reader understands what I am talking about. For short: you did a bad job, then you see a priest and then you hope that all your sins are forgiven and you may continue your job and life without fearing negative consequences and punishment (this is a great concept!). The priest needs food, money, architecture, publishers etc. to build temples, print book and Journals and to reach more clients for his business. And here we sense the very underlying phenomena. Both, the CONCEPTS of religions and industries (economy, nutrition, cars, warfare, architecture, arts, medicine, PUB MED etc.) have been invented by women and men, by our ancestors in order to improve, enrich and increase their power over millions and more millions of people. As such they invented their stories, which serve to fundamentally justify (the ancient Egyptian concept of ma.at) and support their power gaining activities. These beautiful satires include ethics, morals, ideas of gods, angles, saints and afterlife, models of our worlds and our universe: physics, biophysics, biology, mathematics, economy etc. As long as the model works you are successful. When the concept fails, you are out of the game. GAME OVER.

Today we face a fascinating spectrum of phenomena and the reggae goes like this: in the beginning it all has been one single quantum space time molecule of energy, then happened the big bang and it became many, very many. However they all either belonged to religion or to one of the industries (food, engines, fashion, teaching and education etc.). Then came the digital internet and google shaped and fused them into one, and again the world became one single concept: the digital google concept: universal digital reasoning, fate and consciousness. Both, the industry and the religions need, and require the google arena for their existence. Both, all industries and religions depend on the google concept and technology. The most important and fundamental aspect comes next: as google fuses the qualities of the industries (technologies, physics) and religions (morals, ethics), religions and industries start to show their real face, i.e. they are the same (Fig. 2). Now we are allowed to perceive what has been a somehow hidden matter of fact since ever: religions and industries are the same, they are different translators of the will to power, which drives the entire cosmos including our own galaxy. As such google is unique and outstanding as it describes and conducts the fusion of religion and industry (Fig. 2). Google is the moderns master mind and serves both empires: the qualities satisfied by the industries and those satisfied and well take by the religions. Followers become consumers and consumers 
turn into believers etc. Google transforms the world into relicon-valley. Consumerism rules the globe. The users are customers are the believers are the followers (robot surgery, digital oncology). Every touch phones the I, there is no more knowledge, except that published within Wikipedia (Hawaii! and Ancient Greek) for the Medusa Head of the media (ancient Greek only). Every screen follows the follower. Google transforms our societies into the brave and passionate followers of relicon-valley (e reader). All these stars have been invented by women and men, while their kids awaited them in the google kindergarten, silicon valley. Google coined the new appearance and manifestation of the mechanism underlying any perception: "logos ethics the morals" or: the will for power, the will to power and the will to stay in power. Well tubed energy drinks are designed for the will to and of and from the power. Relicon-valley rules the world, coins the world and decides how we translate ourselves into symptoms and signs of consumism. Thus our reggae concludes: it seems to be easy again. It seems to be straight forward again, why to discuss about the differences of religions, politics and industries, economies and other inventions of man? The important message is: all perceptions are translations. Within the matrix of our momental stream of emotions these translations gain their respective value of importance, relevance and truth (Fig. 1). So what? Being is emotion the solution. We all are diluted within the matrix of emotions and atmosphere. This matrix is continuously designed, controlled and orchestrated by the digital master mind of relicon-valley to serve consumerism and will to power (Fig. 1). Taken together, google is digital technology_-based phenomenon, which allows us to sense the real face of religions and technologies. Via google, industries and religions are fused and show their real face, i.e. essentially they are the same (Fig. 2), they are instruments for the translation of will to power into actions serving the increase and maintenance of power.

Lay back and hope that your models continue to work. Trust into your models, as long as they work. Do not over-and under-exist. Do not over and under-do. Harmonise and outbalance your life after this summer and await the things and translations to come.

Going in line with ancient Sumerian, Egyptian and North American Indian myths, man does not die, instead man goes back to the milky way and returns in the form of a star. At first sight the image seems strange. However, modern physics explains how forms of energies transform and translate and that the basis of all sensible and perceivable phenomena are space time quanta. Using the novel interpretation the myth might have sensed that we are all members of the milky way and that we reflect sun light into the milky way and as such we nourish the energies "out there", name it as you will, it all becomes one at the end, at the end of your model, at the end of your reasoning and at the end of your imaginations. As such you may foster the understanding, that we are not as important as we think we are. We are not as exclusive, as we think we are. We are not as unique, as we think we are. If we stop thinking for a moment and listen to the pause depicted at the beginning of the fifth symphony of Ludwig van Beethoven, the essential and fundamental understanding comes to our mind: the very matrix of our collective emotions (logos) manifests (physics) within relicon-valley concept of google. It is full of dreams, hopes, desires and outlooks the skies. May it definitely know it all?

Technology shines bright and fascinates the consumer, thus the consumer may be prone to stay superficial and lack the possibility to sense the underlying emotional matrix of her or his perceptions. Therefore a great surgeon loves robot surgery but will not forget that those who he operates with his robot surgery equipment share fear, anxiety, pain, sorrow and hope. A surgeon always cuts behind the light. Our sun does not serve either day or night, she continuously shines until the matrix takes over. Then all is given back to where it belongs, where the dog ran.

Finally the author declares that he is happy and excited about the great, beautiful, outstanding, positive possibilities fostered by the modern google world. He is aware of the fact how positively google world contributed to the improvement of disease management, diagnosis, therapy, publishing, saving and storage of wisdom, knowledge and myth (sozein; logos). However, maybe the "Google Hupf'" and fire share similar qualities: you may use it for your benefit (improve sight and light), but you may also get burned out and blunted. Therefore, allow the open minded, critical approach and appraisal to academical surgery. Stay tuned, pub med and foster yourself,

\section{Sincerely \\ Martin Riegler}

Acknowledgements The author thanks those who make this project possible: family, friends, colleagues, teachers, patients, publisher, editors, reviewers, authors, readers, seasons, weather, our beautiful globe, moon, stars, galaxies, and the open minded approach searching for the manifestations of the emotional matrix (space time quanta) within others and ourselves.

Conflict of interest M. Riegler declares that he has no competing interests.

Publisher's Note Springer Nature remains neutral with regard to jurisdictional claims in published maps and institutional affiliations. 